\title{
Variables asociadas a la experiencia de abuso en la pareja y su denuncia en una muestra de mujeres*
}

Factors Associated with the Intimate Partner Violence and their Complaint in a Sample of Women

Recibido: mayo 18 de 2012 | Revisado: marzo 1 de 2013 | Aceptado: marzo 9 de 2013

\author{
JuAN CARlos Sierra ** \\ María PAz BERMúdez \\ GUALBERTO BUELA-CASAL \\ José María SALINAS \\ Universidad de Granada, España \\ FredY S. MONGE ${ }^{* * *}$ \\ Universidad Tecnológica de los Andes, Perú
}

doi:10.11144/Javeriana.UPSY13-1.vaea

Para citar este artículo: Sierra, J. C., Bermúdez, M. P., Buela-Casal, G., Salinas, J. M. \& Monge, F. S. (2014). Variables asociadas a la experiencia de abuso en la pareja y su denuncia en una muestra de mujeres. Universitas Psychologica, 13(1), 37-46. doi:10.11144/Javeriana.UPSY13-1.vaea

Artículo de investigación. Agradecimientos: Este estudio fue financiado por la Agencia Española de Cooperación Internacional para el Desarrollo (AECID), dentro del Programa de Cooperación Interuniversitaria e Investigación Científica entre España e Iberoamérica (Proyecto Conjunto de Investigación A/017099/08).

** Universidad de Granada, España. E-mails: jcsierra@ugr.es,maripaz@ugr.es,gbuela@ugr.es,jsalinas@ugr.es

*** Universidad Tecnológica de los Andes, Perú. Email: fred_psi@yahoo.es.
RESUMEN

Se examinan algunos factores asociados a la experiencia de abuso de pareja en la mujer y al hecho de denunciar estos abusos. Se evaluaron la sintomatología psicopatológica, las actitudes sexuales machistas y las variables sociodemográficas en 900 mujeres (300 que habían denunciado a sus parejas por abusos, 300 que nunca lo habían hecho y 300 de la población general), con edades entre 18 y 72 años. El abuso sufrido por la mujer en el ámbito marital se asocia a síntomas psicopatológicos y a actitudes sexuales machistas. La decisión de denunciar a la pareja depende de la intensidad del propio abuso y de que la mujer tenga un rol social más liberal y por tanto menos ajustado a los estándares tradicionales de género.

Palabras clave autores

Abuso de pareja, denuncia, síntomas psicopatológicos, actitudes sexuales machistas, variables sociodemográficas.

Palabras clave descriptores

Violencia familiar, psicología social, psicopatología.

\section{A B S T R ACT}

Some factors associated with intimate partner violence and their complaint among women is here examined. Psychopathological symptoms, sexist attitudes and some sociodemographic variables were assessed in a sample of 900 women (300 women who reported partner abuse, 300 women who never reported partner abuse and 300 women in the general population). The age range was from 18 to 72 years old. The abuse suffered by women within marriage is associated with psychopatological symptoms and sexist attitudes. The women's decision to report partner abuse depends on both the intensity of the abuse and women's values as more liberal and less conformed by traditional gender standards. Keywords authors

Intimate partner violence, complaint, psychopatological symptoms, sexist attitudes, sociodemographic variables.

Keywords plus

Family violence, social psychology, psychopatology. 


\section{Introducción}

Los abusos, tanto psicológicos como físicos, sufridos por la mujer en el ámbito de pareja constituyen un grave problema de salud pública, debido a su incidencia en prácticamente todas las sociedades (Anderson, Ho-Foster, Mitchell, Scheepers \& Goldstein, 2007; García-Moreno, Jansen, Ellsberg, Heise \& Watts, 2006; Loinaz, Ortiz-Tallo, Sánchez \& Ferragut, 2012; Lila, Gracia, \& Murgui, 2013; Novo, Fariña, Seijo \& Arce, 2012; Villafañe Santiago, Jiménez Chafey, De Jesús Carrasquillo \& Vázquez Ramos, 2012) y a los perniciosos efectos que tienen sobre la salud física y mental de las víctimas (Ellsberg, Jansen, Heise, Watts \& García-Moreno, 2008; Gotlieb, 2008; Okuda et al., 2011; PreciadoGavidia, Torres-Cendales \& Rey-Anacona, 2012; Raya Ortega et al., 2004). Se estima que en torno a un tercio de las mujeres de todo el mundo han sido o son víctimas de la violencia de sus parejas (World Health Organization, 2002). En un estudio llevado a cabo por la Organización Mundial de la Salud en diez países de los distintos continentes se concluye que entre el $15 \%$ y el $71 \%$ de mujeres había sido víctima de violencia física o sexual a manos de sus pares (Garcia-Moreno et al., 2006).

De cara a la prevención de este tipo de violencia, es fundamental identificar aquellas variables asociadas que permitan describir y explicar este fenómeno con el fin de desarrollar estrategias de intervención. Se ha centrado el interés en características psicopatológicas de las víctimas que las hiciesen más vulnerables; así, Walton-Moss, Manganello, Frye y Campbell (2005) o Caetano, Vaeth y Ramisetty-Mikler (2008) plantean como un factor de riesgo los problemas de salud mental de la mujer. Se han analizado también ciertos tipos de actitudes; en esta línea, algunos estudios han llegado a demostrar que la intensidad de los abusos de pareja experimentados por las mujeres correlaciona en sentido positivo con sus actitudes sexuales machistas y la actitud favorable hacia la violación (DelgadoÁlvarez, Sánchez Gómez, \& Fernández-Dávila Jara, 2012; Sierra, Costa \& Santos-Iglesias, 2010; Sierra, Monge, Santos-Iglesias, Bermúdez \& Salinas, 2011), presentando la doble moral sexual capacidad para explicar el abuso físico y no físico sufridos (Sierra, Santos-Iglesias \& Gutiérrez-Quintanilla, 2010). Por otro lado, el bajo nivel educativo y la falta de empleo, o empleos no remunerados, se han asociado a la experiencia de abuso en la pareja (Abramsky et al., 2011; Amor, Echeburúa \& Loinaz, 2009; Boy \& Kulczycki, 2008; Cunha \& Abrunhosa Gonçalves, 2013; Echeburúa, Fernández-Montalvo \& Corral, 2008; Estrada Pineda, Herrero Olaizola, \& Rodríguez Díaz, 2012; Sierra, Ortega, GutiérrezQuintanilla, Bermúdez \& Buela-Casal, 2009).

A pesar del elevado número de mujeres que son víctimas de abusos en el ámbito de pareja, son todavía una minoría las que dan el paso de denunciar a sus compañeros sentimentales, especialmente en aquellos países en vías de desarrollo. En un informe del Instituto de la Mujer (1999) se concluye que en España, mientras un 9.2\% de las mujeres encuestadas podría ser considerado "técnicamente" como maltratadas, únicamente un 2.1\% de ellas se describe como tales. Esta discrepancia es mucho más elevada en el reciente estudio de RodríguezFranco et al. (2012) realizado con adolescentes y jóvenes escolarizadas. Tal como indican Ruiz-Pérez, Blanco-Prieto y Vives-Cases (2004), esta diferencia podría deberse a que la mujer acepta o considera como algo natural padecer ciertos tipos de abusos al interior de la pareja, lo que dificultaría o imposibilitaría su denuncia.

No obstante, la sociedad en general, y la mujer en particular, es cada día más consciente de la necesidad de denunciar estos delitos. Así, por ejemplo, en España desde enero de 2007 a junio de 2010 las denuncias experimentaron un incremento del 17\% (Consejo General del Poder Judicial, 2010). Por esto, también es relevante, de cara a desarrollar estrategias de intervención, identificar las características personales de aquellas mujeres que deciden denunciar a su pareja por abusos, que las hacen diferentes de aquellas otras que sufriendo abusos no se deciden a dar ese paso.

Por tanto, el objetivo de este estudio es determinar qué factores se asocian a la experiencia de abuso en la mujer en el ámbito de pareja y al hecho de denunciar estos abusos; así, se examinará el papel 
que desempeñan la psicopatología, las actitudes sexuales machistas (doble moral sexual y actitud favorable hacia la violación) y algunas variables sociodemográficas (edad, tipo de relación de pareja, nivel de estudios, ocupación laboral y pertenencia a asociaciones de mujeres).

\section{Método}

\section{Participantes}

La muestra del estudio está compuesta por 900 mujeres peruanas, seleccionadas mediante un procedimiento incidental entre la población general de la Provincia del Cuzco. Sus edades están comprendidas entre 18 y 72 años $(M=31.99 ; D E=$ 8.97). La mayoría de ellas mantenía una relación heterosexual desde hacía al menos seis meses. Un 62.6\% vivía con su pareja, casi la mitad tenía estudios superiores (48.2\%), aproximadamente un tercio estaba constituido por amas de casa (31\%), un poco más de la mitad trabajaba fuera del hogar $(51.2 \%)$ y un $25.5 \%$ pertenecía a alguna asociación de mujeres (Tabla 1). Del total de 900 mujeres, 300

\section{TABLA 1}

Características sociodemográficas de las mujeres

\begin{tabular}{lc}
\hline & $N(\%)$ \\
\hline Relación de pareja & \\
$\quad$ Sí & $711(79.6)$ \\
No & $182(20.4)$ \\
\hline Tipo de relación & \\
Pareja sin convivencia & $262(29.3)$ \\
Pareja con convivencia & $560(62.6)$ \\
En trámites de separación & $36(4)$ \\
Separada & $36(4)$ \\
\hline Nivel de estudios & \\
Sin estudios & $49(5.4)$ \\
Educación Primaria & $132(14.7)$ \\
Educación Secundaria & $285(31.72)$ \\
Estudios Superiores & $434(48.2)$ \\
\hline Ocupación laboral & \\
Estudiante & $159(17.7)$ \\
Ama de casa & $278(31)$ \\
Trabajo que no requiere estudios superiores & $202(22.5)$ \\
Trabajo que requiere estudios superiores & $257(28.7)$ \\
\hline Pertenece a alguna asociación de mujeres & \\
$\quad$ Sí & $227(25.5)$ \\
$\quad$ No & $664(74.5)$ \\
\hline
\end{tabular}

Fuente: elaboración propia. habían denunciado a sus parejas por abusos, 300 no habían llevado a cabo nunca ninguna denuncia al respecto y de las otras 300 se desconocía esta circunstancia.

Instrumentos

- Cuestionario sociodemográfico con el que se recabó información acerca de las características sociodemográficas recogidas en la Tabla 1.

- Versión española del Index of Spouse Abuse ([ISA]; Hudson \& McIntosh, 1981) de Sierra et al. (2011). Formado por 19 ítems agrupados en dos dimensiones: Abuso no físico $(\alpha=0.93)$ y Abuso físico $(\alpha=0.89)$. Los ítems son contestados en una escala tipo Likert desde 1 (nunca) hasta 5 (casi siempre) de modo que muestran la frecuencia de abusos al interior de la pareja. Se ha establecido un punto de corte de 13 para Abuso no físico y de 8 para Abuso físico (Sierra et al., 2011). En la muestra de este estudio se obtuvieron coeficientes de fiabilidad de consistencia interna de 0.93 para Abuso no físico y 0.9 para Abuso físico.

- Versión peruana de la Double Standard Scale ([DSS]; Caron, Davis, Halteman \& Stickle, 1993) de Sierra, Monge, Santos-Iglesias, Rodríguez y Aparicio (2010) compuesta por 9 items contestados en una escala tipo Likert de 5 puntos, desde 1 (totalmente en desacuerdo) a 5 (totalmente de acuerdo), los cuales permiten evaluar el grado de aceptación de la doble moral sexual tradicional. Su fiabilidad de consistencia interna es de 0.79; en la muestra de este estudio se obtuvo un coeficiente de 0.86 .

- Versión peruana de la Rape Supportive Atittude Scale ([RSAS]; Lottes, 1991) de Sierra, Monge et al. (2010) formada por 20 ítems contestados en una escala tipo Likert de 5 puntos desde 1 (muy en desacuerdo) hasta 5 (muy de acuerdo). Evalúa las actitudes favorables al uso de la violencia del hombre hacia la mujer en el contexto de las relaciones sexuales. La fiabilidad de consistencia interna es de 0.87 ; en la muestra de este estudio se obtuvo un coeficiente alfa de Cronbach de 0.88 . 
- Cuestionario de 90 Síntomas SCL-90-R (Derogatis, 2002) formado por 90 síntomas valorados en una escala de 0 (ausencia total de molestias relacionadas con el síntoma) a 4 (molestia máxima), proporcionando nueve dimensiones sintomáticas de psicopatología y tres índices globales de malestar. La fiabilidad de consistencia interna oscila entre 0.81 y 0.9 , y la fiabilidad test-retest a una semana se sitúa entre 0.78 y 0.9. En el presente estudio se tuvieron en cuenta las nueve dimensiones sintomáticas cuyos coeficientes de fiabilidad de consistencia interna fueron: Somatización $(\alpha=0.89)$, Obsesión-compulsión ( $\alpha=0.87$ ), Sensibilidad interpersonal ( $\alpha=0.85)$, Depresión $(\alpha=0.9)$, Ansiedad ( $\alpha=0.88)$, Hostilidad ( $\alpha=0.82)$, An- siedad fóbica ( $\alpha=0.85$ ), Ideación paranoide ( $\alpha$ $=0.8)$ y Psicoticismo $(\alpha=0.89)$.

\section{Procedimiento}

La evaluación de la muestra seleccionada de forma incidental se realizó en la Provincia de Cuzco (Perú), a lo largo del año 2009, de forma individual o en pequeños grupos (asociaciones de mujeres, centros de trabajo, docentes y asistenciales), por medio de tres evaluadores entrenados para tal fin. Las mujeres que habían denunciado abuso fueron evaluadas en instituciones públicas asistenciales de este tipo de violencia familiar, fundamentalmente en la Fiscalía de la Familia. Todas las participantes recibieron las mismas instrucciones, asegurándose en todo momento el carácter voluntario de su parti-

TABLA 2

Variables en la ecuación de regresión logística que predice el abuso no físico

\begin{tabular}{|c|c|c|c|c|c|}
\hline & $\begin{array}{l}\text { Sin abuso no } \\
\text { físico }\end{array}$ & $\begin{array}{l}\text { Con abuso no } \\
\text { físico }\end{array}$ & OR & \multicolumn{2}{|c|}{ IC 95\% } \\
\hline Ansiedad & $7.80(5.82)^{b}$ & $17.85(8.28)^{b}$ & $2.104^{* *}$ & 1.333 & 3.323 \\
\hline Hostilidad & $5.19(3.93)^{b}$ & $10.96(5.18)^{b}$ & $1.677^{* *}$ & 1.205 & 2.333 \\
\hline Ansiedad fóbica & $4.99(4.42)^{\mathrm{b}}$ & $12.34(6.21)^{b}$ & $1.729^{* *}$ & 1.14 & 2.621 \\
\hline Actitud favorable hacia la violación & $43.01(10.67)^{b}$ & $53.6(13.29)^{b}$ & $1.032^{* *}$ & 1.015 & 1.049 \\
\hline Sin relación ${ }^{a}$ (porcentaje) & $126(28)$ & $51(11.8)$ & & & \\
\hline Con relación (porcentaje) & $323(71.9)$ & $381(88.2)$ & $1.82^{*}$ & 1.123 & 2.95 \\
\hline $\begin{array}{l}\text { Pareja sin } \\
\text { convivencia }{ }^{\text {(porcentaje) }}\end{array}$ & $169(37.6)$ & $87(20.1)$ & & & \\
\hline $\begin{array}{l}\text { Convivencia } \\
\text { en pareja (porcentaje) }\end{array}$ & $256(57)$ & $299(69.2)$ & 1.162 & 0.751 & 1.798 \\
\hline $\begin{array}{l}\text { Trámites de } \\
\text { Separación (porcentaje) }\end{array}$ & $9(2)$ & $26(6)$ & $8.404^{* *}$ & 2.998 & 23.563 \\
\hline Separación (porcentaje) & $15(3.3)$ & $20(4.6)$ & $3.536^{* *}$ & 1.406 & 8.894 \\
\hline $\begin{array}{l}\text { Ama de } \\
\text { casa }^{a} \text { (porcentaje) }\end{array}$ & $84(18.7)$ & $193(44.7)$ & & & \\
\hline Estudiante (porcentaje) & $72(16)$ & $79(18.3)$ & 0.694 & 0.397 & 1.215 \\
\hline Trabajo sin estudios superiores (porcentaje) & $110(24.5)$ & $90(20.8)$ & $0.484^{* *}$ & 0.299 & 0.783 \\
\hline Trabajo con estudios superiores (porcentaje) & $183(40.8)$ & $70(16.2)$ & $0.42^{* *}$ & 0.254 & 0.695 \\
\hline Constante & & & $0.018^{* *}$ & & \\
\hline
\end{tabular}

Nota. ${ }^{\text {a }}$ categoría de referencia; ${ }^{\mathrm{b}}$ media (DE). ${ }^{*} p<0.05 .{ }^{* *} p<0.01$.

Fuente: elaboración propia. 
cipación, así como el anonimato y confidencialidad de todas las respuestas dadas. Antes de comenzar a responder a las diferentes pruebas, todas las mujeres otorgaron su consentimiento informado verbal.

\section{Resultados}

En primer lugar, se identificó la prevalencia de abuso. Teniendo en cuenta los puntos de corte del ISA establecidos por Sierra et al. (2011) se detectó abuso no físico en 432 mujeres (48\%) y abuso físico en 333 (37\%).

En segundo lugar, se examinó qué variables se asociaban a cada uno de los tipos de abuso. Tal como muestra la Tabla 2, el modelo final de regresión logística asoció el Abuso no físico con las variables ansiedad, hostilidad, ansiedad fóbica, actitud favorable hacia la violación, tener pareja y estar separada o en trámites de separación; por su parte, tener un trabajo que no requiera estudios superiores o que lo requiera se asocia negativamente con el Abuso no físico. Teniendo en cuenta, los tres tipos de variables analizadas (psicopatología, actitudes y variables sociodemográficas), las que presentan una mayor asociación son la ansiedad (OR: 2.1; IC 95\%: 1.33-3.32), la actitud favorable hacia la violación (OR: 1.03; IC 95\%: 1.01-1.04) y estar en trámites de separación (OR: 8.4; IC 95\%: 2.99-23.56).

La Tabla 3 presenta el modelo final de regresión logística en el que el Abuso físico se asoció con ansiedad, ansiedad fóbica, psicoticismo, actitud favorable hacia la violación y con tener relación de pareja, siendo la asociación más importante la de la ansiedad fóbica (OR: 2.68; IC 95\%: 1.76-4.07).

En tercer lugar, se planteó identificar las variables asociadas al hecho de denunciar a la pareja por abusos. Entre las 600 mujeres de las que se tenía información acerca de si habían presentado denuncia o no por malos tratos de sus parejas (300 mujeres de cada circunstancia), el ISA determinó que 292 (48.67\%) habían sufrido abuso no físico, de las que 208 (71.2\%) habían presentado denuncia. En el modelo de regresión logística se asoció con la presentación de denuncia la puntuación en Abuso físico del ISA, ansiedad fóbica, doble moral sexual, actitud favorable hacia la violación y pertenecer a una organización femenina, asociándose negativamente tener un trabajo que no requiera estudios superiores (Tabla 4). Las asociaciones más intensas se dan con la ansiedad fóbica (OR: 3.26; IC 95\%: 1.56-6.82), la doble moral sexual (OR: 1.37; IC 95\%: 1.24-1.51) y el pertenecer a una asociación de mujeres (OR: 2.95; IC 95\%: 1.04-8.32).

Asimismo, entre estas 600 mujeres, el ISA estableció que 236 (39.33\%) habían experimentado abuso físico, habiendo presentado denuncia 184 (78\%). En la ecuación de regresión logística se asociaron positivamente con la presentación de denuncia las variables ansiedad fóbica, doble moral sexual y edad, asociándose negativamente

TABLA 3

Variables en la ecuación de regresión logística que predice el abuso físico

\begin{tabular}{lccccc}
\hline & Sin abuso físico & Con abuso físico & \multirow{2}{*}{ OR } & \multicolumn{2}{c}{ IC 95\% } \\
\hline Ansiedad & $8.5(6.36)^{\mathrm{b}}$ & $19.63(7.64)^{\mathrm{b}}$ & $1.799^{*}$ & 1.109 & 2.918 \\
Ansiedad fóbica & $5.39(4.74)^{\mathrm{b}}$ & $13.84(5.55)^{\mathrm{b}}$ & $2.682^{* *}$ & 1.766 & 4.075 \\
Psicoticismo & $8.54(7.1)^{\mathrm{b}}$ & $19.59(7.65)^{\mathrm{b}}$ & $1.576^{*}$ & 1.059 & 2.343 \\
Actitud favorable hacia la violación & $43.91(10.95)^{\mathrm{b}}$ & $55.21(13.42)^{\mathrm{b}}$ & $1.04^{* *}$ & 1.021 & 1.058 \\
Sin relación $^{\mathrm{a}}$ (porcentaje) & $148(26.7)$ & $33(9.9)$ & & & \\
Con relación (porcentaje) & $406(73.3)$ & $300(90.1)$ & $1.754^{*}$ & 1.06 & 2.902 \\
Constante & & & $0.004^{* *}$ & & \\
\hline
\end{tabular}

Nota. ${ }^{\text {a }}$ categoría de referencia; ${ }^{\mathrm{b}}$ media $(\mathrm{DE}) ;{ }^{*} p<0.05 .{ }^{* *} p<0.01$.

Fuente: elaboración propia. 
TABLA 4

Variables en la ecuación de regresión logística que predice la presentación de denuncia entre las mujeres que sufren abuso no físico

\begin{tabular}{lccccc}
\hline & $\begin{array}{c}\text { Mujeres que no } \\
\text { denuncian } \\
n=84\end{array}$ & $\begin{array}{c}\text { Mujeres que } \\
\text { denuncian } \\
n=208\end{array}$ & OR & \multicolumn{2}{c}{ IC 95\% } \\
\hline Abuso físico & $9.69(4.5)^{\mathrm{b}}$ & $14.99(5.81)^{\mathrm{b}}$ & $1.157^{* *}$ & 1.036 & 1.291 \\
Ansiedad fóbica & $7.36(5.08)^{\mathrm{b}}$ & $14.11(5.2)^{\mathrm{b}}$ & $3.266^{* *}$ & 1.562 & 6.829 \\
Doble moral sexual & $19.13(5.79)^{\mathrm{b}}$ & $28.92(6.47)^{\mathrm{b}}$ & $1.371^{* *}$ & 1.24 & 1.517 \\
Actitud favorable hacia la violación & $45.66(12.39)^{\mathrm{b}}$ & $56.21(13.18)^{\mathrm{b}}$ & $1.055^{*}$ & 1.013 & 1.1 \\
Ama de casa (porcentaje) $^{\text {a }}$ & $21(25)$ & $125(60.1)$ & & & \\
Estudiante (porcentaje) & $9(10.7)$ & $35(16.8)$ & 2.196 & 0.463 & 10.426 \\
Trabajo sin estudios universitarios (porcentaje) & $19(22.6)$ & $33(15.9)$ & $0.177^{* *}$ & 0.055 & 0.565 \\
Trabajo con estudios universitarios (porcentaje) & $35(41.7)$ & $15(7.2)$ & 0.348 & 0.088 & 1.377 \\
No pertenecer a asociación de mujeres & $70(83.3)$ & $94(45.2)$ & & & \\
(porcentaje) & $14(16.7)$ & $114(54.8)$ & $2.954^{*}$ & 1.049 & 8.322 \\
Pertenecer a asociación de & & & 0 & & \\
Mujeres (porcentaje) & & & & & \\
Constante & & & & & \\
\hline
\end{tabular}

Nota. ${ }^{\text {a }}$ categoría de referencia; ${ }^{\mathrm{b}}$ media $(\mathrm{DE}) ;{ }^{*} \mathrm{p}<0.05 .{ }^{* *} p<0.01$.

Fuente: elaboración propia.

TABLA 5

Variables en la ecuación de regresión logística que predice la presentación de denuncia entre las mujeres que sufren abuso físico

\begin{tabular}{lccccc}
\hline & $\begin{array}{c}\text { Mujeres que no } \\
\text { denuncian } \\
n=52\end{array}$ & $\begin{array}{c}\text { Mujeres que } \\
\text { denuncian } \\
n=184\end{array}$ & OR & \multicolumn{2}{c}{ IC 95\% } \\
\hline Ansiedad fóbica & $8.67(5.6)^{\mathrm{b}}$ & $14.58(5.06)^{\mathrm{b}}$ & $1.278^{* *}$ & 1.152 & 1.419 \\
Doble moral sexual & $19.57(6.27)^{\mathrm{b}}$ & $28.50(6.56)^{\mathrm{b}}$ & $1.242^{* *}$ & 1.14 & 1.353 \\
Edad & $30.06(7.12)^{\mathrm{b}}$ & $35.44(10.03)^{\mathrm{b}}$ & $1.081^{*}$ & 1.013 & 1.152 \\
Ama de casa (porcentaje) & $10(19.2)$ & $114(62)$ & & & \\
Estudiante (porcentaje) & $7(13.5)$ & $28(15.2)$ & 0.819 & 0.183 & 3.658 \\
Trabajo sin estudios superiores (porcentaje) & $13(25)$ & $30(16.3)$ & $0.153^{* *}$ & 0.048 & 0.49 \\
Trabajo con estudios superiores (porcentaje) & $22(42.3)$ & $12(6.5)$ & $0.079^{* *}$ & 0.018 & 0.338 \\
Constante & & & 0 & & \\
\hline
\end{tabular}

Nota. ${ }^{a}$ categoría de referencia; ${ }^{b} \operatorname{media}(D E) ;{ }^{*} p<0.05 .{ }^{*} p<0.01$.

Fuente: elaboración propia.

tener un trabajo que no requiere estudios superio-

res y si el trabajo requiere estudios superiores ( $\mathrm{Ta}$ -

bla 5). En este caso las asociaciones más intensas

son también con la ansiedad fóbica (OR: 1.27; IC

95\%: 1.15-1.41) y doble moral sexual (OR: 1.24;

IC 95\%: 1.14-1.35).

\section{Discusión}

El primer resultado relevante que se extrae de este estudio es el elevado porcentaje de mujeres evaluadas que son víctimas de abusos dentro de la pareja. El $48 \%$ que padece abuso no físico y el $37 \%$ con abuso físico son valores superiores al $30.01 \%$ y $20.1 \%$ de mujeres salvadoreñas (Sierra et al., 
2009) o al 29.59\% y $7.37 \%$ de mujeres brasileñas (Costa, Sierra, Bermúdez \& Buela-Casal, 2009), respectivamente. Estos dos últimos estudios, al emplear el mismo instrumento de evaluación del abuso, aunque no utilicen un punto de corte, sino el porcentaje medio de abuso en todos los ítems, permiten hacer una comparativa entre muestras de distintos países. Estos datos evidencian la relevancia del problema, pues llegan a superar el $34.2 \%$ de abuso físico y sexual que informan García-Moreno et al. (2006) en la misma zona geográfica.

El segundo aspecto abordado fue determinar qué variables psicopatológicas, actitudinales y sociodemográficas se asocian a la experiencia de abuso dentro de la pareja. La ansiedad, una actitud favorable hacia la violación y el hecho de mantener una relación de pareja desde al menos seis meses constituyen factores importantes a la hora de predecir tanto el abuso no físico como el físico. Estudios previos habían ya señalado la asociación de la experiencia de abuso de pareja con trastornos de ansiedad (Blasco-Ros, Sánchez-Lorente \& Martínez, 2010; Loxton, Schofield \& Hussain, 2006; Preciado-Gavidia et al., 2012) y actitudes favorables hacia la violación (Sierra, Monge et al., 2010; Sierra et al., 2011) o violencia marital (Vizcarra Larrañaga \& Póo Figueroa, 2011). En cambio, otras variables analizadas aparecen asociadas de forma específica a un tipo u otro de abuso. Así, el abuso no físico es predicho, además de por las dos variables señaladas, por la hostilidad, una relación en proceso o en estado de separación y por el trabajo fuera del hogar; en este último caso, la asociación es de tipo negativo.

Por lo que respecta al abuso físico, se incluye como variable predictora específica el psicoticismo. De este modo, en cuanto a las dimensiones psicopatológicas, parece demostrarse que la mujer abusada por su pareja acaba desarrollando sentimientos y conductas propias de un estado de agresividad, ira, irritabilidad, resentimiento (Dutton, 1995; Kuijpers, van der Knaap \& Willem Winkel, 2011) y alienación social (Bonomi et al., 2006). Las variables sociodemográficas relacionadas con el estado marital y la ocupación laboral únicamente predicen el abuso no físico, asociándose este a parejas separadas o en proceso de separación (Amor, Echeburúa, de Corral, Zubizarreta \& Sarasua, 2002; Brownridge, 2006; Sierra et al., 2009), mientras que el hecho de trabajar fuera del hogar se asociaría a un menor riesgo de padecer abusos (Sierra et al., 2009; Stöckl, Heise \& Watts, 2011).

En tercer lugar, se trató de identificar aquellas variables asociadas al hecho de que la mujer denuncie o no los abusos. Es conocido que un gran porcentaje de casos de abuso no llegan nunca a ser denunciados, por lo que es fundamental determinar que características diferenciales presentan aquellas mujeres que se deciden a dar este paso. Una primera cuestión que llama la atención es que entre las mujeres que se pueden catalogar como abusadas según el punto de corte establecido para el ISA, hay un porcentaje importante de ellas que no denuncian el problema: un $28.8 \%$ de mujeres con abusos psicológicos y un $22 \%$ con abusos físicos. Ello pone de manifiesto que no siempre la mujer abusada toma la decisión de denunciar a su pareja, bien sea por falta de conciencia de abuso (Ruiz-Pérez et al., 2004) o por otras razones, como el miedo o la presión social. Si se analizan las variables asociadas al hecho de denunciar, se aprecia que es la mayor intensidad y cronicidad del abuso (reflejada en forma de abusos físicos, niveles altos de ansiedad, presencia de actitudes sexuales machistas y mayor edad), así como el hecho de pertenecer a asociaciones de mujeres y trabajar fuera de casa, las que llevan a la mujer abusada a denunciar a su pareja. Estos datos ponen de manifiesto que, desgraciadamente, se tiende a denunciar cuando el abuso es más intenso y crónico, pero también dejan entrever que la liberación de la mujer en forma de pertenencia a asociaciones femeninas y su inserción en el mercado laboral constituyen hechos que posibilitan que las mujeres tomen conciencia del problema que padecen.

En definitiva, los resultados de este estudio evidencian claramente que el abuso sufrido por la mujer en el ámbito marital constituye un grave problema de salud asociado a síntomas psicopatológicos y a la presencia de actitudes sexuales machistas. Por su parte, la decisión de denunciar a la pareja depende de la intensidad del propio abuso, pero también del hecho de que la mujer tenga un 
rol social más liberal y por tanto menos ajustado a los estándares tradicionales de género.

\section{Referencias}

Abramski, T., Watts, C. H., García-Moreno, C., Devries, K., Kiss, L. \& Ellsberg, M. (2011). What factors are associated with recent intimate partner violence? Findings from the WHO multi-country study on women's health and domestic violence. BMC Public Health, 11(109), 1-17.

Amor, P. J., Echeburúa, E., Corral, P., Zubizarreta, I. \& Sarasua, B. (2002). Repercusiones psicopatológicas de la violencia doméstica en la mujer en función de las circunstancias del maltrato. Revista Internacional de Psicología Clínica y de la Salud, 2(2), 227-246.

Amor, P. J., Echeburúa, E. \& Loinaz, I. (2009). ¿Se puede establecer una clasificación tipológica de los hombres violentos contra su pareja? International Journal of Clinical and Health Psychology, 9(3), 519-539.

Anderson, N., Ho-Foster, A., Mitchell, S., Scheepers, E. $\&$ Goldstein, S. (2007). Risk factors for domestic physical violence: National cross-sectional household surveys in eight southern African countries. BMC Women Health, 7(11), 1-13.

Blasco-Ros, C., Sánchez-Lorente, S. \& Martínez, M. (2010). Recovery from depressive symptoms, state anxiety and post-traumatic stress disorder in women exposed to physical and psychological, but not to psychological intimate partner violence alone: A longitudinal study. BMC Psychiatry, 10(98), 1-12.

Bonomi, A. E., Thompson, R. S., Anderson, M., Reid, R. J., Carrell, D., Dimer, J. A. \& Rivara, F. P. (2006). Intimate partner violence and women's physical, mental, and social functioning. American Journal of Preventive Medicine, 30(6), 458-466.

Boy, A. \& Kulczycki, A. (2008). What we know about intimate partner violence in the Middle East and North Africa. Violence Against Women, 14(1), 53-70.

Brownridge, D. A. (2006). Violence against women post-separation. Aggression and Violent Behavior, 11(5), 514-530.

Caetano, R., Vaeth, P. A. \& Ramisetty-Mikler, S. (2008). Intimate partner violence victim and perpetrator characteristics among couples in United States. Journal of Family Violence, 23(6), 507-518.
Caron, S. L., Davis, C. M., Halteman, W. A. \& Stickle, M. (1993). Double Standard Scale. En C. M. Davis, W. L. Yarber, R. Bauserman, G. Scherer \& S. L. Davis (Eds.), Handbook of sexuality-related measures (pp. 182-183). Londres: Sage.

Consejo General del Poder Judicial. (2010). Balance positivo tras cinco años de ley integral contra la violencia de género (Informe). Madrid: Observatorio contra la violencia doméstica y de género. Recuperado el 10 de noviembre de 2011, de http://www. observatorioviolencia.org/upload_images/File/ DOC1290772991_OVG_Informe_5_anos.pdf

Costa, N., Sierra, J. C., Bermúdez, M. P. \& Buela-Casal, G. (2009). Comparación entre mujeres blancas y negras víctimas de la violencia de pareja en el nordeste de Brasil. Revista Colombiana de Psicología, 18(2), 135-143.

Cunha, O. \& Abrunhosa Gonçalves, R. (2013). Intimate partner violence offenders: Generating a data based typology of batterers and implications for treatment. The European Journal of Psychology Applied to Legal Context, 5(2), 131-139.

Delgado-Álvarez, M. C., Sánchez Gómez, M. C., \& Fernández-Dávila Jara, P. A. (2012). Atributos y estereotipos de género asociados al ciclo de la violencia contra la mujer. Universitas Psychologica, 11(3), 769-777.

Derogatis, L. R. (2002). SCL-90-R. Cuestionario de 90 sintomas. Madrid: TEA.

Dutton, D. G. (1995). A scale for measuring propensity for abusiveness. Journal of Family Violence, 10(2), 203-221.

Echeburúa, E., Fernández-Montalvo, J. \& Corral, P. (2008). ¿Hay diferencias entre la violencia grave y la violencia menos grave contra la pareja? International Journal of Clinical and Health Psychology, 8(2), 355-382.

Ellsberg, M., Jansen, H. A. F. M, Heise, L., Watts, C. H. \& Garcia-Moreno, C. (2008). Intimate partner violence and women's physical and mental health in the WHO multi-country study on women's health and domestic violence: An observational study. Lancet, 371(9619), 1165-1172.

Estrada Pineda, C., Herrero Olaizola, J. \& Rodríguez Díaz, F. (2012). La red de apoyo en mujeres víctimas de violencia contra la pareja en el estado de Jalisco 
(México). La red de apoyo en mujeres víctimas de violencia contra la pareja en el estado de Jalisco (México). Universitas Psychologica, 11(2), 523-534.

Garcia-Moreno, C., Jansen, H. A. F. M., Ellsberg, M., Heise, L. \& Watts, C. H. (2006). Prevalence of intimate partner violence: Findings from the WHO multi-country study on women's health and domestic violence. Lancet, 368(9543), 1260-1269.

Gotlieb, A. S. (2008). Intimate partner violence: A clinical review of screening and intervention. Women's Health, 4(5), 529-539.

Instituto de la Mujer (1999). La violencia contra las mujeres. Resultados de la macroencuesta. Madrid: Ministerio de Trabajo y Asuntos Sociales e Instituto de la Mujer.

Hudson, W. W. \& McIntosh, S. R. (1981). The assessment of spouse abuse: Two quantifiable dimensions. Journal of Marriage and the Family, 43(4), 873-888.

Kuijpers, K. F., van der Knaap, L. M. \& Willem Winkel, F. (2011). Risk of revictimization of intimate partner violence: The role of attachment, anger and violent behavior of the victim. Journal of Family Violence, 26(7), 545-549. doi: 10.1007/s10896011-9399-8

Lila, M., Gracia, E., Murgui, S. (2013). Psychological adjustment and victim-blaming among intimate partner violence offenders: The role of social support and stressful life events. The European Journal of Psychology Applied to Legal Context, 5(2), 147-153.

Loinaz, I., Ortiz-Tallo, M., Sánchez, L. M. \& Ferragut, M. (2011). MCMI-III Grossman personality facets among partner-violent men in prison. International Journal of Clinical and Health Psychology, 12(3), 389404.

Lottes, I. L. (1991). Belief systems: Sexuality and rape. Journal of Psychology and Human Sexuality, 4(1), 37-59.

Loxton, D., Schofield, M. \& Hussain, R. (2006). Psychological health in midlife among women who have ever lived with a violent partner or spouse. Journal of Interpersonal Violence, 21(8), 1092-1107.

Novo, M., Fariña, F., Seijo, M. D., \& Arce, R. (2012). Assessment of a community rehabilitation programme in convicted male intimate-partner violence offenders. International Journal of Clinical and Health Psychology, 12(2), 219-234.
Okuda, M., Olfson, M., Hasin, D., Grant, B. F., Link, K. H. \& Blanco, C. (2011). Mental health of victims of intimate partner violence: Results from a national epidemiologic survey. Psychiatric Services, 62(8), 959-962.

Preciado-Gavidia, P. M., Torres-Cendales, N. E. \& ReyAnacona, C. A. (2012). Mujeres que finalizaron una relación maltratante: características de personalidad, psicopatológicas y sociodemográficas. Universitas Psychologica, 11(1), 43-54.

Raya Ortega, L., Ruiz Pérez, I., Plazaola Castaño, J., Brun López-Abisab, S., Rueda Lozano, D., García de Vinuesa, L., et al. (2004). Intimate partner violence as a factor associated to health problems. Atención Primaria, 34(3), 117-127.

Rodríguez-Franco, L., López-Cepero Borrego, J., Rodríguez-Díaz, F. J., Estrada Pineda, C., Antuña Bellerín, M. A. \& Quevedo-Blasco, R. (2012). Labeling dating abuse: Undetected abuse among Spanish adolescents and young adults. International Journal of Clinical and Health Psychology, 12(1), 55-67.

Ruiz-Pérez, I., Blanco-Prieto, P. \& Vives-Cases, C. (2004). Violencia contra la mujer en la pareja: determinantes y respuestas sociosanitarias. Gaceta Sanitaria, 18(Supl.), 4-12.

Sierra, J. C., Costa, N. \& Santos-Iglesias, P. (2010). Un estudio acerca de las propiedades psicométricas de la versión brasileña del Index of Spouse Abuse. Revista Iberoamericana de Diagnóstico y Evaluación Psicológica, 2(30), 57-70.

Sierra, J. C., Monge, F. S., Santos-Iglesias, P., Bermúdez, M. P. \& Salinas, J. M. (2011). Validation of a reduced Spanish version of the Index of Spouse Abuse. International Journal of Clinical and Health Psychology, 11(2), 363-383.

Sierra, J. C., Monge, F. S., Santos-Iglesias, P., Rodríguez, K. \& Aparicio, D. (2010). Propiedades psicométricas de las versiones en español de la Double Standard Scale (DSS) y de la Rape Supportive Scale (RSAS) en mujeres peruanas. Cuadernos de Medicina Psicosomática y Psiquiatría de Enlace, 95, 57-66.

Sierra, J. C., Ortega, V., Gutiérrez-Quintanilla, J. R., Bermúdez, M. P. \& Buela-Casal, G. (2009). Violencia de pareja en mujeres salvadoreñas. Prevalencia y factores sociodemográficos de riesgo. Revista Argentina de Clínica Psicológica, 18(2), 115-123. 
Sierra, J. C., Santos-Iglesias, P. \& Gutiérrez-Quintanilla, J. R. (2010). Validación del Índice de Abuso en la Pareja en mujeres de El Salvador. Revista Mexicana de Psicología, 27(1), 5-14.

Stöckl, H., Heise, L. \& Watts, C. (2011). Factors associated with violence by a current partner in a nationally representative sample of German women. Sociology of Health and Illness, 33(5), 694-709.

Villafañe Santiago, A. A., Jiménez Chafey, M. I., De Jesús Carrasquillo, D. \& Vázquez Ramos, R. A. (2012). Desarrollo del Cuestionario de Experiencias de Violencia en las Relaciones de Pareja y Familia en estudiantes universitarios. Universitas Psychologica, 11(1), 207-215.

Vizcarra Larrañaga, M. B. \& Póo Figueroa, A. M. (2011). Violencia de pareja en estudiantes universitarios del sur de Chile. Universitas Psychologica, 10(1), 89-98.

Walton-Moss, B. J., Manganello, J., Frye, V. \& Campbell, J. C. (2005). Risk factors for intimate partner violence and associated injury among urban women. Journal of Community Health, 30(5), 377-389.

World Health Organization. (2002). Violence by intimate Partners. En WHO (Eds.), World report on violence and health (pp. 89-121). Ginebra: Autor. 